\title{
Investigation of Secondary Neutron Production in Large Space Vehicles for Deep Space
}

\author{
Kristina Rojdev, ${ }^{1}$ Steve Koontz, ${ }^{2}$ and Brandon Reddell ${ }^{3}$ \\ NASA Johnson Space Center, Houston, TX 77058 \\ William Atwell ${ }^{4}$ \\ Retired Boeing Technical Fellow, Houston, TX 77058 \\ and \\ Paul Boeder ${ }^{5}$ \\ Boeing Research and Technology, Houston, TX 77058
}

\begin{abstract}
Future human spaceflight programs will focus on deep space, asteroids, lunar, and Mars surface operations with larger, more massive spacecraft structures necessary for transportation of crew and cargo. In addition to the challenges of manufacturing and launching these larger, more massive structures, there are added challenges from the space radiation environment and its impacts on the crew, electronics, and vehicle materials. Primary radiation from the sun (solar particle events) and from outside the solar system (galactic cosmic rays) interact with materials of the vehicle and the elements inside the vehicle. These interactions lead to the primary radiation being absorbed with production of secondary radiation (primarily pions, nuclear fragments, and neutrons). With all vehicles, the highenergy primary radiation is of most concern. However, with larger, more massive vehicles, there are more opportunities for secondary radiation production in the larger spacecraft effective shielding thickness, which can be significant enough to cause concern. In a previous paper $^{1}$, we embarked upon our first steps toward studying neutron production in more massive vehicles by validating our radiation transport codes for neutron environments against flight data from the Space Shuttle, Mir, and the International Space Station. The following paper will extend the previous work to focus on the deep space environment and the resulting secondary neutron flux internal to vehicles that are more massive.
\end{abstract}

\section{Nomenclature}

$\begin{array}{ll}G C R & =\text { Galactic Cosmic Radiation } \\ G e V & =\text { Gigaelectron volt } \\ G L E & =\text { Ground Level Event } \\ H Z E T R N & =\text { High Charge and Energy Transport code } \\ L E O & =\text { low Earth orbit } \\ S P E & =\text { Solar Particle Event }\end{array}$

\footnotetext{
${ }^{1}$ Aerospace Engineer, Systems Engineering \& Test Branch, 2101 NASA Parkway/MC: EA531, AIAA Member.

${ }^{2}$ International Space Station System Manger for Space Environments, 2101 NASA Parkway/MC: ES4, AIAA Member.

${ }^{3}$ Avionics Systems Division, 2101 NASA Parkway/MC: EV5, non-member

${ }^{4}$ Retired, 16623 Park Green Way, AIAA Associate Fellow.

${ }^{5}$ Now with JPL: Aerospace Engineer, Propulsion, Thermal and Materials Engineering Section, MC: 125-103, 4800 Oak Grove Dr, Pasadena, CA, non-member.
}

American Institute of Aeronautics and Astronautics 


\section{Introduction}

TASA is developing new vehicles for future missions to deep space LaGrange points, asteroids, and eventually Mars. These vehicles tend to be much larger than previous vehicles developed during the manned space era because the crew will need more logistics to survive the longer missions. Given the increase in size, we wanted to investigate the consequences of these increased shielding thicknesses in worst-case, deep space radiation environments.

In a previous paper ${ }^{1}$, we investigated a typical ISS orbit of $362.5 \mathrm{~km}$ and an inclination of $51.6^{\circ}$ that included the GCR exposure, trapped protons from the Van Allen radiation belts, and secondary albedo neutron data. A variety of thicknesses $\left(0.1,0.5,1.0,5.0,10.0,20.0,50.0,100.0,150.0\right.$, and $\left.200.0 \mathrm{~g} / \mathrm{cm}^{2}\right)$ of aluminum were studied to represent the potential vehicle sizes that might be employed. Our simulations of the resulting neutron flux were then compared against flight data taken from the ISS, Shuttle, and Mir missions. The results from that study showed that our simulations were in good agreement with neutron flight data at the vehicle thicknesses available, thus validating our simulations. In addition, our observations showed that there was an overall increase in neutron flux with increasing vehicle shielding thickness.

This study will build upon the previous study and extend it to the deep space environment. Thus, we are looking at worst-case galactic cosmic radiation (GCR) and solar particle event (SPE) environments, two different material configurations, and multiple thicknesses to understand secondary neutron production for these cases.

\section{Background}

\section{A. Radiation Environment}

For human spaceflight, the type of radiation of interest is generally the primary radiation, which is radiation that has not interacted with another material. However, as spacecraft designs and concepts have gotten larger and more massive to accommodate longer human missions, the influence of secondary radiation also needs consideration. Secondary radiation, such as neutrons, is radiation produced from primary radiation interacting with spacecraft materials. The following section discusses these types of radiation in more detail.

\section{Primary Radiation}

During a traverse to Mars or during a mission at a libration point, for example, a vehicle will be in what is known as "deep space." This part of our solar system has two primary forms of radiation that can interact with the vehicle. Solar particle events (SPEs) are streams of energetic particles, primarily protons, which originate from the sun. Large spacecraft can generally shield the crew and electronics from these events. However, extremely energetic SPEs, such as Ground Level Events (GLEs), ${ }^{2}$ are cause for concern with large, heavily shielded spacecraft due to potential secondary radiation production. Two examples of GLEs are in Figure 1, which shows the integral and differential energy spectra for the series of SPEs that occurred during 19-24 October 1989 and the "Bastille Day" event that occurred on 14 July 2000.

American Institute of Aeronautics and Astronautics 


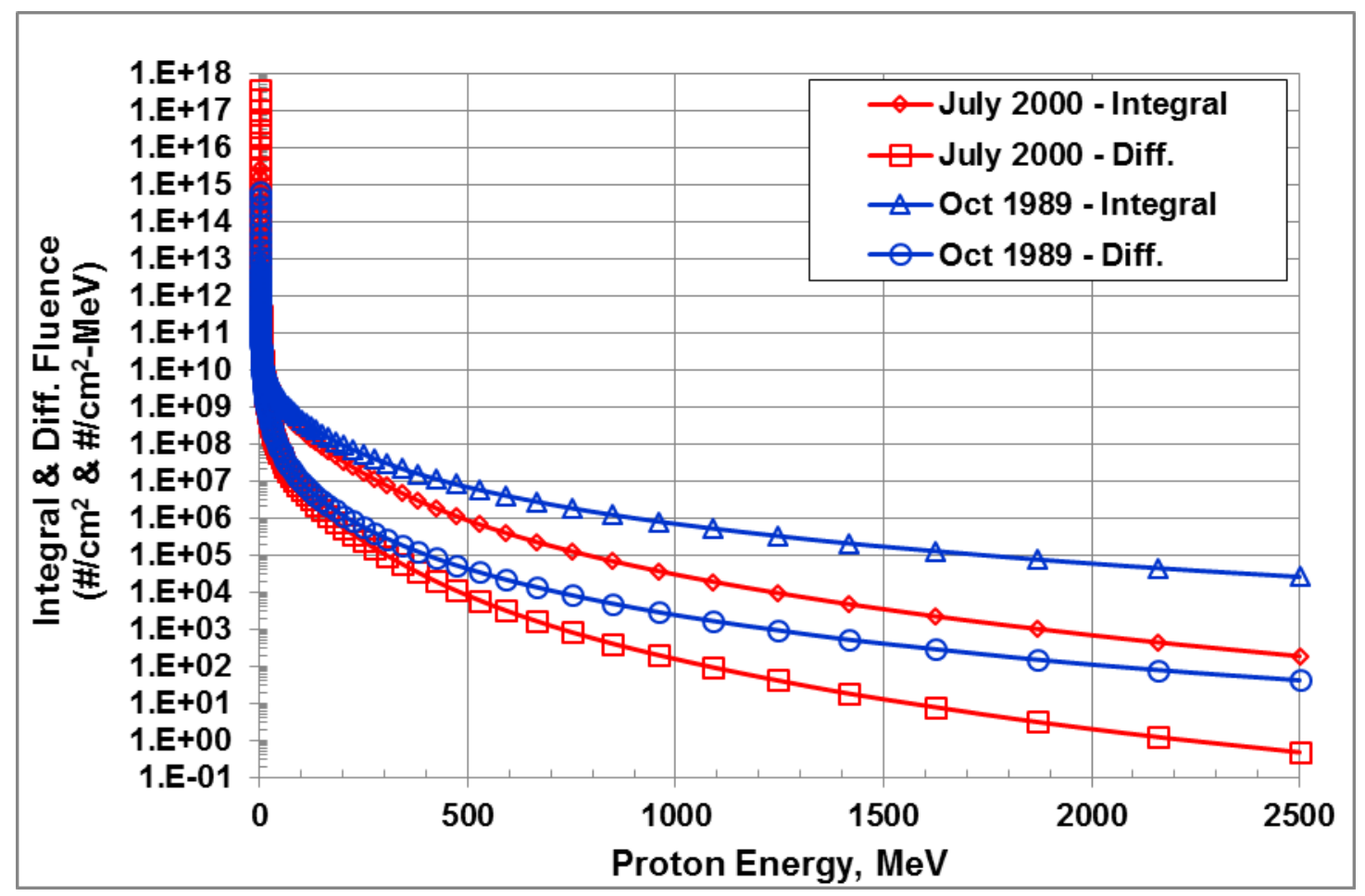

Figure 1: Band fit integral and differential energy spectra for two extremely large SPEs that occurred on October 1989 and 14 July 2000. From 19-24 October 1989, there were three GLEs and an energetic solar particle (ESP), and the energy spectra shown in the plot is a combination of these four events.

Another form of primary radiation experienced in deep space is galactic cosmic radiation (GCR). These particles range from protons to heavy ions, such as iron, and are extremely energetic, extending into the GeV energy range. Given the energy of GCRs, large spacecraft cannot easily shield them and they tend to produce a significant amount of secondary radiation. Figure 2 is a plot of the GCR energy spectra for several elemental species for both solar minimum and solar maximum.

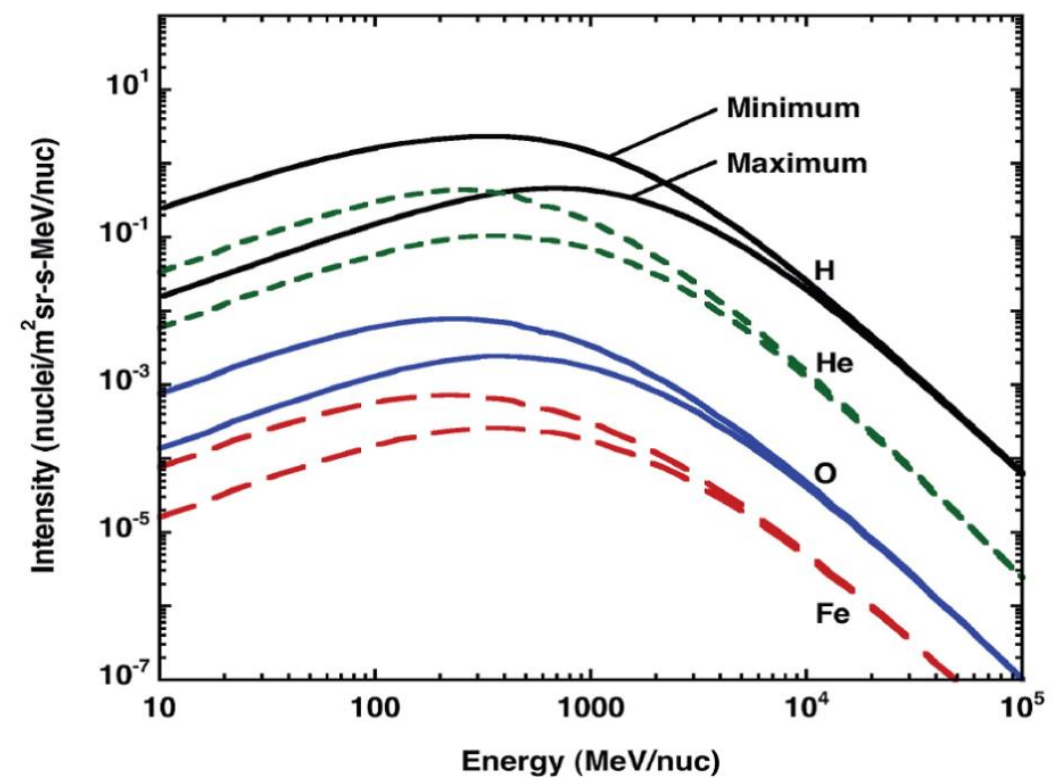

Figure 2: Differential flux of several GCR elemental species $(\mathrm{H}, \mathrm{He}, \mathrm{O}$, and Fe) at solar minimum and solar maximum conditions. $^{3}$

American Institute of Aeronautics and Astronautics 


\section{Secondary Radiation}

These sources of primary radiation interact with materials (the vehicle, electronics, humans, etc.) in deep space, leading to the production of secondary radiation. Both forms of primary radiation contain charged particles. Low energy charged particles primarily cause ionization in a target material, leading to additional ionizations within the target due to delta rays. However, with high-energy charged particles, particularly with the heavy ions in GCRs, nuclear interactions also occur. These nuclear interactions lead to changes in the nucleus of the target or fragmentation of both target and primary nuclei, thus producing the secondary radiation from the material ${ }^{4}$.

\section{B. Transport Code}

To keep consistent with our previous work, we used the high charge and energy transport code, HZETRN2010. . $^{5-6}$ This code was developed at NASA Langley Research Center and is a one dimensional, deterministic transport code that uses a straight ahead approximation of the Boltzmann transport equation for charged and neutral particles, with a continuous slowing down approach.

\section{Method}

The environments selected for study were based on worst-case scenarios for deep space missions. Thus, we used the 1977 solar minimum GCR environment, which has a high fluence of GCR particles, and the October 1989 Band fit solar particle event, which was a GLE-type event.

We simulated two vehicle configurations. The first is an aluminum vehicle to provide a comparison with the work completed in our previous paper. The second is a vehicle that comprises aluminum with cargo. For the second vehicle, we assume $69 \%$ of the depth is from the cargo and $31 \%$ of the depth is from aluminum. In addition, we assume the following items for cargo $28.7 \%$ water, $17.5 \%$ food, $8.1 \%$ oxygen, $0.4 \%$ nitrogen, $8.5 \%$ crew provisions, $5.5 \%$ water container, $17.8 \%$ oxygen tank, $3 \%$ nitrogen tank, $3.4 \%$ crew provision container, $4.4 \%$ cargo transfer bags, and $2.7 \%$ human waste container.

For both of these configurations, we primarily considered thicknesses representative of typical spacecraft at 20 $\mathrm{g} / \mathrm{cm}^{2}$, thicknesses of ISS-sized spacecraft of $50 \mathrm{~g} / \mathrm{cm}^{2}$, and large space vehicles that might be developed for deep space and Mars missions of $100 \mathrm{~g} / \mathrm{cm}^{2}$. However, we also investigated thicknesses of 10, 150, and $200 \mathrm{~g} / \mathrm{cm}^{2}$.

\section{Results and Discussion}

The neutron fluxes behind the three primary thicknesses we are investigating of aluminum and aluminum with cargo are plotted in Figure 3. When comparing the SPE and GCR environment at a specific thickness, we see similar behavior in the neutron flux of the two vehicle configurations. At $20 \mathrm{~g} / \mathrm{cm}^{2}$, the neutron flux for a vehicle with aluminum and cargo is slightly higher than one of all aluminum at very low energies, but at higher energies become comparable. At $50 \mathrm{~g} / \mathrm{cm}^{2}$, the neutron flux of the two vehicles are comparable for most of the energy spectrum, with aluminum producing slightly more neutrons at the lower energies. At $100 \mathrm{~g} / \mathrm{cm}^{2}$, an aluminum vehicle has a higher neutron flux than a vehicle of aluminum and cargo for energies below $100 \mathrm{MeV}$. Furthermore, in all cases, the solar particle event generates more secondary neutrons than the GCR environment. However, the SPE is a singular event that occurs over a short duration, whereas the neutron flux from GCR is the flux over one day. Thus, depending on mission length, the GCR flux can produce more secondary radiation than the single SPE. It is also important to note that the neutron kinetic energy spectrum produced in primary space radiation interactions with spacecraft materials is very different from Earth surface nuclear reactor or radioisotope decay environments. The very high-energy neutrons seen in the secondary space radiation environment are not present to the same extent in the Earth surface environments, but are present because of GCR interactions with Earth's atmosphere. ${ }^{7}$

American Institute of Aeronautics and Astronautics 


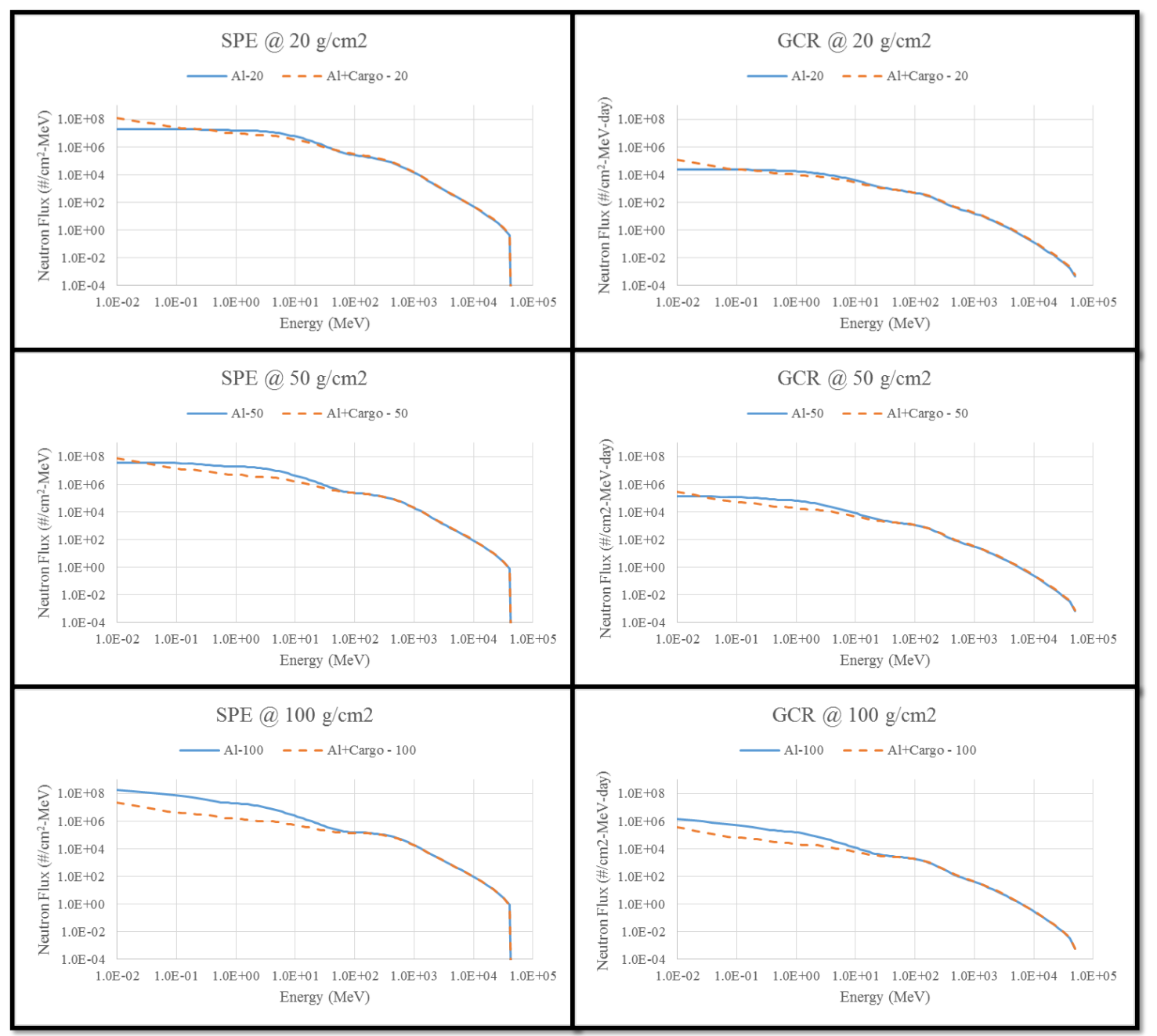

Figure 3: Comparison of neutron fluxes for an all-aluminum vehicle and a vehicle with aluminum and cargo. The plots on the left hand side are for the SPE environment and the plots on the right hand side are for the GCR environment. The thicknesses considered are 20,50 , and $100 \mathrm{~g} / \mathrm{cm}^{2}$, respectively, from the top to the bottom.

Another way to visualize this data is to integrate over the energy levels using the trapezoidal rule (Equation 1). The integrated data is then plotted against thickness to better understand how the neutron flux changes with increasing shielding mass (Figure 4). We also plot the integrated flux for the International Space Station (ISS), from our previous paper ${ }^{1}$, for comparison.

$$
\text { Integrated Flux }=\sum\left(X_{i}-X_{i-1}\right)\left(\frac{f\left(X_{i-1}\right)+f\left(X_{i}\right)}{2}\right)
$$

From this visualization, it is clear that the deep space environment generates more secondary neutrons in a spacecraft than in low Earth orbit (LEO). The neutron flight data from the ISS was gathered at around $50 \mathrm{~g} / \mathrm{cm}^{2}$. In deep space, a vehicle with the same effective shielding mass as ISS would produce approximately an order of magnitude greater number of neutrons in one day, from the GCR alone.

It is also apparent that there are more neutrons produced from the solar particle event than there are from one day in the GCR environment. For those electronics that may be susceptible to neutron exposure, it may be prudent to consider when the mission is to take place to limit the probability of encountering a GLE type SPE. However, the GCR environment used in this study is from epoch solar minimum 1977. Recent solar predictions show an overall decline in solar activity, and increasing GCR activity, up to an increase in intensity of approximately $25 \%{ }^{8-10}$. Therefore, this GCR case may not represent an accurate worst-case scenario for future solar minimum conditions.

For further discussion, if we consider a Mars mission in which the vehicle transits to Mars, orbits Mars for a period of time, and then returns to Earth, we can make the following comparisons. Assuming the vehicle is similar in size

American Institute of Aeronautics and Astronautics 
$\left(50 \mathrm{~g} / \mathrm{cm}^{2}\right)$ and material (aluminum) to ISS, has a mission length of approximately three years, occurs during a deep solar minimum, and happens to encounter one GLE SPE (a worst case scenario), the interior of the vehicle will accumulate a neutron fluence of $1.17 \mathrm{E} 9$ neutrons $/ \mathrm{cm}^{2}$. The ISS over three years will accumulate $9.01 \mathrm{E} 7$ neutrons $/ \mathrm{cm}^{2}$. Thus, vehicle materials, electronics, and crew on a Mars mission will essentially receive approximately thirteen times more neutron exposure than they would in low Earth orbit, over a three-year period. Future work should focus on understanding the implications of the additional neutron exposure for these deep space missions.

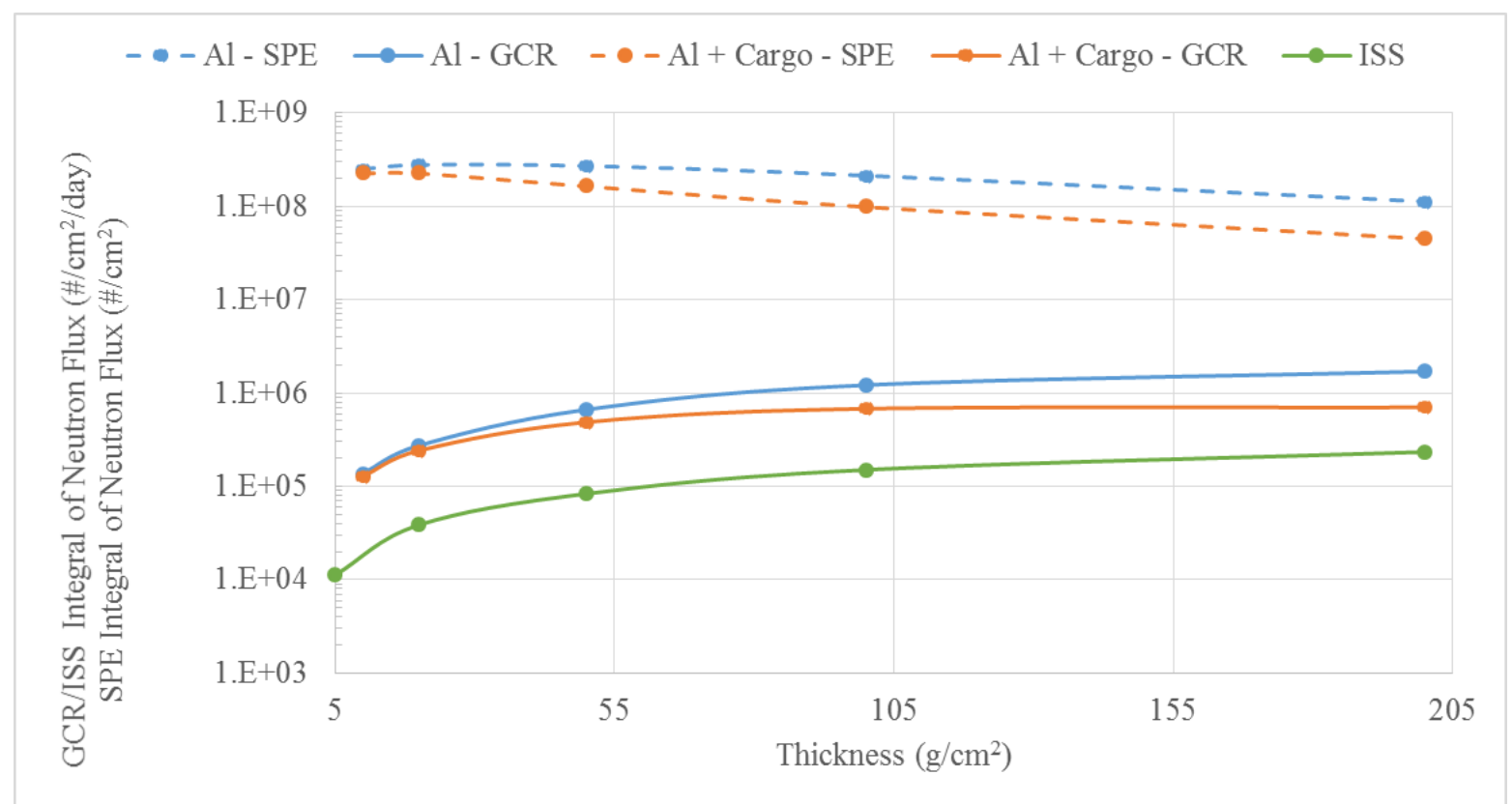

Figure 4: Integrated neutron flux of the two vehicle configurations and the two environments against thickness of shielding mass. The lines in blue represent the aluminum vehicle configuration, whereas the lines in orange represent the aluminum and cargo vehicle configuration. The dashed lines represent the SPE environment and the solid lines represent the GCR environment. Note that the SPEs are specific events of limited duration while the GCR are continuously present. Thus, if we integrate over a full year, the GCR secondary flux will meet or exceed the SPE secondary neutron flux.

We also see in the data that in both environments, the aluminum configuration produces more neutrons than the aluminum and cargo configuration. In our previous study, we showed that the all-aluminum configuration matched well with the International Space Station, Shuttle, and Mir. Thus, if future vehicles focus on limiting the amount of aluminum in the structure and increasing the amount of cargo for shielding, or alternatively changing the material of the structure to comprise lower-Z elements, then the production of neutrons due to the vehicle mass will decrease.

Furthermore, in the SPE environment, there is a decreasing trend in the number of neutrons with increasing thickness, whereas in the GCR environment, there is an increasing trend in the number of neutrons with increasing thickness. Therefore, for a GLE type SPE, having larger vehicles seems beneficial, but the likelihood of encountering a GLE type SPE needs to be considered. If the probability of this type of SPE is minimal, then increasing the shielding to reduce neutron production may be more detrimental when you consider the GCR environment, which is a more likely scenario of the two. However, for the Aluminum and cargo vehicle in the GCR environment, after $100 \mathrm{~g} / \mathrm{cm}^{2}$, there does not seem to be an appreciable increase in the neutron production with increasing vehicle thickness.

\section{Summary and Conclusions}

This study investigated two potential vehicle configurations for deep space and the worst-case primary radiation environments in which they may operate. The results were also compared with our previous study of the neutron environment inside the ISS. We found that deep space radiation causes larger neutron production than when in a low Earth orbit environment. We also found that a GLE type solar particle event produces more neutrons than one day in a GCR environment during solar minimum. However, depending on mission length, the neutron production due to a GCR environment will meet or exceed that of the SPE environment. We further found that the aluminum configuration produces more neutrons than the aluminum and cargo configuration. Finally, we found that neutron production increases with shielding thickness in the GCR case, but decreases with shielding thickness in the SPE case. 
Thus, we conclude that changing materials in the vehicle to increase the amount of low- $\mathrm{Z}$ materials will be beneficial in reducing the number of neutrons produced. Increasing vehicle thickness in a GCR environment, particularly during a deep solar minimum condition, may lead to increased neutron production to a point. If low-Z materials are used in the vehicle design over aluminum, there may not be appreciable additional neutron production after thicknesses of approximately $100 \mathrm{~g} / \mathrm{cm}^{2}$. Future work will extend this analysis to a more recent GCR environment to better determine the neutron production resulting from the deep solar minimum conditions currently being experienced, as well as to understand the implications of the higher neutron production in deep space when compared with LEO.

\section{References}

${ }^{1}$ Rojdev, K., Koontz, S, Reddell, B, Atwell, W., and Boeder, P., "Comparison and Validation of FLUKA and HZETRN as Tools for Investigating the Secondary Neutron Production in Large Space Vehicles," Proceedings of AIAA SPACE Conference, Pasadena, CA, 2015.

${ }^{2}$ Tylka, A., Dietrich, W., and Atwell, W., "Band Function Representations of Solar Proton Spectra in Ground-Level Events," 38th Scientific Assembly of the Committee on Space Research (COSPAR), Bremen, Germany, 2010.

${ }^{3}$ Badhwar, G.D., Cucinotta, F.A., and O’Neill, P.M., "An analysis of interplanetary space radiation exposure for various solar cycles," Radiation Research, 138(2), 201-208, 1994.

${ }^{4}$ Tribble, A. C., The Space Environment: Implications for Spacecraft Design, Princeton University Press, Princeton, 2003, Ch. 5.

${ }^{5}$ Slaba, T.C., Blattnig, S.R., Badavi, F.F., "Faster and more accurate transport procedures for HZETRN," Journal of Computational Physics, 229, 9397-9417, 2010a.

${ }^{6}$ Slaba, T.C., Blattnig, S.R., Aghara, S.K., Townsend, L.W., Handler, T., Gabriel, T.A., Pinsky, L.S., Reddell, B., "Coupled Neutron Transport for HZETRN," Radiation Measurements, 45, 173-182, $2010 \mathrm{~b}$.

${ }^{7}$ Sato, T., Niita, K., "Analytical Functions to Predict Cosmic Ray Neutron Spectra in the Atmosphere," Radiation Research, $166(3), 544-555,2006$.

${ }^{8}$ Mewaldt, R.A., David, A.J., Lave, K.A., Leske, R.A., Stone, E.C., Wiedenbeck, M.E., Binns, W.R., Christian, E.R., Cummings, A.C., de Nolfo, G.A., Israel, M.H., Labrador, A.W., von Rosenvinge, T.T., "Record-Setting Cosmic Ray Intensities in 2009 and 2010," The Astrophysical Journal Letters, 723 (1), L1, 2010.

${ }^{9}$ Krainev, M.B., "On the Intensity of Galactic Cosmic Rays in the Inner Heliosphere during Epochs of Minimum Solar Activity," Bulleting of the Lebedev Physics Institute, 39 (6), 185-190, 2012.

${ }^{10}$ McDonald, F.B., Webber, W.R., Reames, D.V., "Unusual Time Histories of Galactic and Anomalous Cosmic Rays at 1 AU Over the Deep Solar Minimum of Cycle 23/24," Geophysical Research Letters, 37 (18), 2010.

American Institute of Aeronautics and Astronautics 